\title{
Incidence of infectious complications following transrectal ultrasound-guided prostate biopsy in Calgary, Alberta, Canada: A retrospective population-based analysis
}

\author{
Jan Krzysztof Rudzinski, MD; Jun Kawakami, MD, FRCSC ${ }^{\dagger}$ \\ *Faculty of Medicine, University of Calgary, Calgary, AB; ‘Southern Alberta Institute of Urology, Calgary, AB
}

Cite as: Can Urol Assoc J 2014;8(5-6):e301-5. http://dx.doi.org/10.5489/cuaj.1751 Published online May 21, 2014.

\section{Abstract}

Introduction: We have seen an increased risk of infectious complications following transrectal ultrasound-guided prostate biopsy (TRUS-PB). Fluoroquinolone (FQ) antibiotics are common for prophylaxis prior to TRUS-PB. We evaluate whether increasing FQ resistance correlates with increased incidence of post-biopsy infectious complications at our institution.

Methods: We conducted a retrospective chart and electronic health record review on 927 patients who underwent TRUS-PB between January and July of 2012 in Calgary, Alberta, Canada. We prospectively collected the following variables: age, pre-biopsy prostatespecific antigen, and date of biopsy. We documented presentation to an emergency department within 30 days of TRUS-PB for infectious and non-infectious complications.

Results: Of the 927 patients, 58 patients $(6.3 \%)$ were admitted to the emergency department due to post-TRUS-PB complications within 30 days post-biopsy. The most common infectious complications were sepsis in 21 patients $(2.2 \%)$, followed by urinary tract infection (UTI) in $9(0.9 \%)$, and prostatitis in $4(0.4 \%)$. We found that $83 \%$ of the septic episodes and $66.6 \%$ of the UTIs were attributed to ciprofloxacin resistant Escherichia coli (E. coli). The incidence of non-infectious complications was as follows: urinary retention in $12(1.2 \%)$, hematuria in $9(0.9 \%)$, and rectal bleeding in $8(0.8 \%)$.

Conclusion: Our results suggest an increased incidence of infectious complications caused by FQ resistant organisms following TRUS-PB. This finding could be attributed to increasing community resistance to ciprofloxacin. The current antimicrobial prophylactic regimen needs to be re-evaluated, and a novel approach may need to be considered.

\section{Introduction}

There is a rising incidence of infectious complications after transrectal ultrasound-guided prostate biopsy (TRUS-PB) in North America and Europe. ${ }^{1-4}$ This increase may be due to the global rise in antibacterial resistance. ${ }^{3}$ Infectious com- plications occur in about $1 \%$ to $6 \%$ of patients undergoing prostate biopsy. The most common complications include sepsis, urinary tract infection (UTI), prostatitis, and epididymo-orchitis. ${ }^{1,5-7}$ Post-biopsy infections are frequently associated with colonic fluoroquinolone (FQ) resistant Escherichia coli (E. coli) inoculated from the rectum into the urinary tract. ${ }^{8}$

The current American and Canadian Urological Association Guidelines recommend FQ antibiotics as firstline agents prior to TRUS-PB. ${ }^{9,10}$ Randomized clinical control trials have demonstrated efficacy of $F Q$ in reducing the incidence of post-TRUS-PB sepsis. ${ }^{11,12}$ However, despite the reported effectiveness of $F Q$, we are currently observing a global rise in bacterial resistance to these agents., ${ }^{1,4,5}$ About $11 \%$ to $22 \%$ of males undergoing TRUS-PB harbour FQ resistant bacteria within their rectum. ${ }^{13-17}$

Regional reports from our site demonstrate a rise in ciprofloxacin resistance in community strains of $\mathrm{E}$. coli from $7 \%$ to $11 \%$ over the past 5 years. ${ }^{18,19}$ In Latin America and India, the rates are as high as $75 \% .^{20,21}$ The global spread of the E. coli sequence type (ST131) with multiple antimicrobial resistance genes and the horizontal transfer gene are of great concern in the development of resistance to prophylactic antibiotics. ${ }^{22}$ Patient-specific risk factors for FQ resistance include increasing age, comorbidities, international travel, recent hospitalization, prior FQ exposure and urinary catheterization. ${ }^{1,3,23,24}$

The aim of our study was to characterize the prescribing patterns of prophylactic antibiotics prior to TRUS-PB in a large urban centre. We also investigate the relationship between increasing $\mathrm{E}$. coli resistance rates and the incidence of infectious complications following prostate biopsy. More importantly, we aim to stimulate discussion around the potential modification of clinical practice guidelines for pre-biopsy antimicrobial prophylaxis.

\section{Methods}

Ethics approval for the study was granted by the Chair of Conjoint Health Research Ethics Board of the University of 
Calgary. We conducted a retrospective chart and electronic health record review of 927 patients who underwent TRUSPB between January and July of 2012 at the Southern Alberta Institute of Urology (SAIU).

Pre-biopsy prescribing patterns among urologists at our institution was determined by the results of a survey inquiring about first- and second-line antibiotics prior to TRUS-PB. In addition, we inquired about the influence of patient-specific risk factors on the choice of the pre-biopsy antibacterial regimen.

All 12-core TRUS-PB were performed by radiologists at the Prostate Cancer Centre at the SAIU in Calgary, Alberta, Canada. All patients received a prescription for a 3-day course of oral antibiotic prior to undergoing their scheduled TRUS-PB. We only included patients who were scheduled to undergo their first prostate biopsy.

The variables collected prospectively included patient age, pre-biopsy prostate-specific antigen (PSA), and the date of TRUS-PB. Since previous studies have demonstrated that most serious infectious complications occur within 30 days of TRUS-PB, we documented the presentation to the emergency department and the ambulatory care centre within 1 month of the biopsy.

The variables collected retrospectively included length of stay in the hospital, need for intensive care (ICU) admission, as well as blood and urine culture results, which were then analyzed for bacterial susceptibility and resistance to antibiotics. The sensitivities and resistance rates are presented as percentages of the number of samples tested for each antibiotic.

\section{Results}

According to the survey results at the SAIU, the most common first-line agent for prophylaxis was the 3-day regimen of oral ciprofloxacin. Second-line antibiotics were oral trimetroprim-sulfamethoxazole or cephalexin. All patients were advised to take their 3-day antibiotic regimen as follows: 1 day prior, the day of, and 1 day post-biopsy. According to our survey, 8 of 15 urologists indicated the following risk factors: prior exposure to FQ within 3 months of the scheduled biopsy, travel history to areas with a high incidence of FQ resistance, as well as close contact with relatives who travelled to regions with high rates of $\mathrm{FQ}$ resistance.

Over the 7-month study interval, we identified 927 patients (average age: 61.6 years) who underwent TRUS-PB at our institution. Overall, $58(6.3 \%)$ patients were admitted to the emergency department 30 days post-TRUS-PB. We recorded a total of $71(7.6 \%)$ complications: $40(4.3 \%)$ infectious and 31 (3.3\%) non-infectious (Fig. 1).

The most commonly observed infectious complications were sepsis in 21 patients $(2.2 \%)$, followed by UTI in 9 $(0.9 \%)$, prostatitis in $4(0.4 \%)$ and fever in $2(0.2 \%)$. There was 1 episode of peri-anal abscess, scrotal wall abscess, urethritis, and epididymo-orchitis in each of the remaining 4 patients with post-biopsy infections. Out of 9 isolated cases of UTI, 6 urine samples $(66.6 \%)$ were ciprofloxacin-resistant E. coli. The most prevalent non-infectious complication was urinary retention in 12 patients $(1.2 \%)$, followed by hematuria in $9(0.9 \%)$, and rectal bleeding in $8(0.8 \%)$.

The average septic patient was 60 years old and admitted 2.23 days post-TRUS-PB. The definition of sepsis in our analysis is the presence of documented or suspected infection with at least 2 criteria for systemic inflammatory response syndrome (SIRS). ${ }^{25}$

Of the 21 cases of sepsis, 18 cases were blood culture positive and 3 cases were blood culture negative. These 3 patients had a suspected infection and satisfied the SIRS criteria on admission to the emergency department. In addition, 13 of the septic patients had identical blood and urine culture results with identical antibacterial susceptibilities. The remaining 5 patients had positive blood culture results with a negative urine cultures (Fig. 2). The most common bacteria responsible for sepsis was E.coli. On average, septic patients were admitted for 5.1 days. There were no ICU admissions for patients admitted with sepsis.

Blood isolates were most resistant to ciprofloxacin, ampicillin, and trimetroprim-sulfamethoxazole, with resistance rates of $85.7 \%, 71.4 \%$, and $50 \%$, respectively (Table 1 ). Susceptibility was greatest to gentamicin at $78.6 \%$, piperacillin-tazobactam at $78.5 \%$, and amoxicillin-clavulin at $71.4 \%$. Table 2 depicts the overall susceptibility and resistance rates of organisms isolated from the blood of patients admitted for sepsis. The highest resistance is noted to ciprofloxacin, ampi-

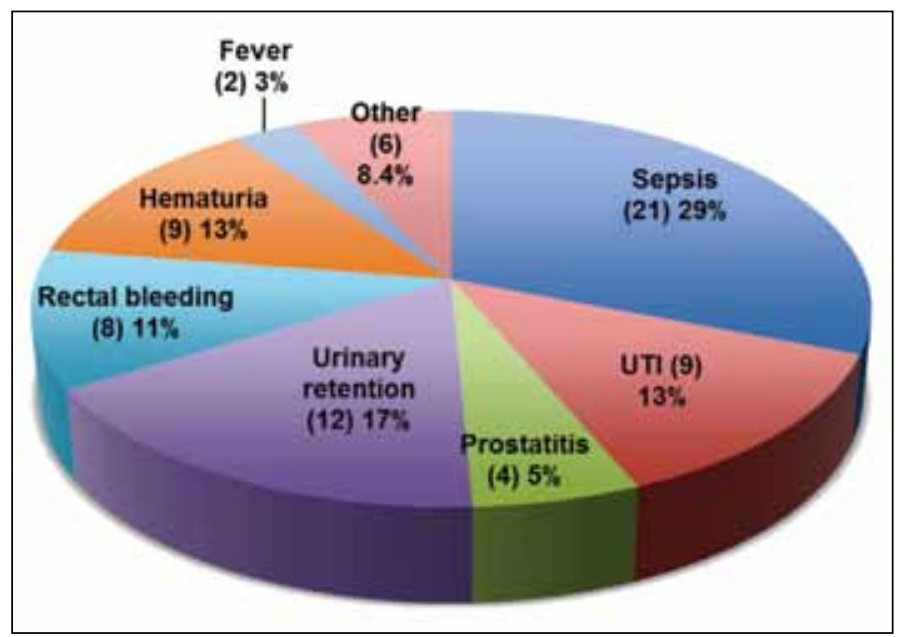

Fig. 1. Overall distribution of post-transrectal ultrasound-guided prostate biopsy complications. This figure depicts the distribution of 71 complications in 58 patients who presented to the emergency department. Out of 71 cases, $56.3 \%$ were due to infections and $43.7 \%$ due to non-infectious causes. The "Other" category contains 6 of the following separate complications: peri-anal abscess, scrotal wall abscess, urethritis, epididymo-orchitis, urinary frequency, and gross hematuria with clots. 


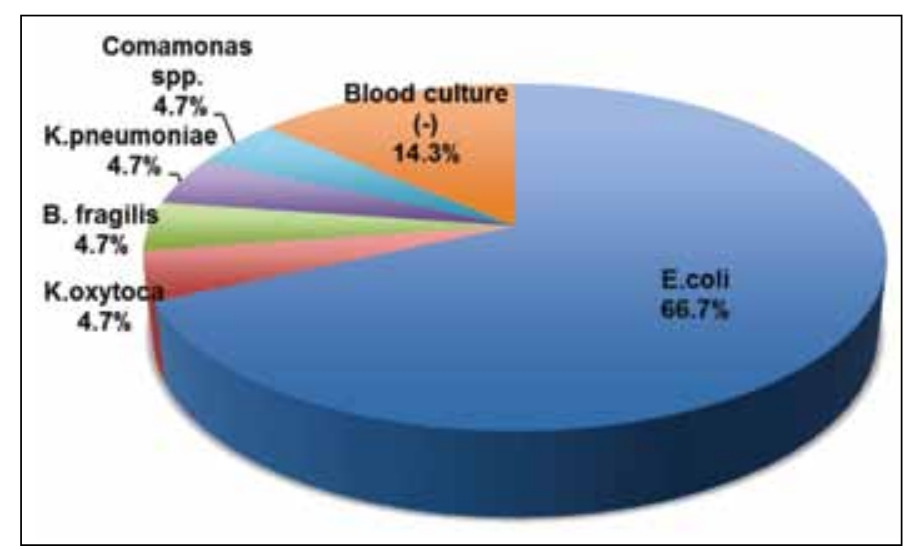

Fig. 2. Distribution of microorganisms responsible for sepsis. Out of 21 total cases of sepsis, 18 patients were blood culture positive and 3 patients had no growth in their blood. E. coli was responsible for 14 cases of sepsis and remaining 4 cases were due to each of the following: Klebisella pneumonia, Klebsiella oxytoca, Bacterioides fragilis, and Comamonas species.

cillin, norfloxacin, and TMP-SMX with rates of $87.5 \%, 80 \%$, $50 \%$, and $47.1 \%$, respectively. Susceptibility was greatest to piperacillin-tazobactam $(100 \%)$, amoxicillin-clavulin $(85.7 \%)$, and gentamicin $(82.4 \%)$.

\section{Discussion}

Prostate cancer is the second most common cancer in men worldwide and most commonly diagnosed cancer among men in Canada. ${ }^{26}$ The 12 -core TRUS-PB is the established gold standard modality for histological prostate cancer diagnosis. Currently, it is estimated that about 1 million TRUS-PB are performed on annually in North America., ${ }^{3,27}$ The increasing incidence of hospitalization due to post-biopsy complications are a barrier to prostate cancer detection and active surveillance of patients with low-risk prostate cancer. ${ }^{27}$ The microbes responsible for most infectious complications at our centre reveal high resistance rates to the antibiotics recommended by North American Clinical Practice Guidelines.

Currently, the global incidence of post-TRUS-PB sepsis is between $0.1 \%$ and $5.0 \% .^{1,2,27}$ The rates at our institution are closer to the upper limit of the global range, with $83 \%$ of septic episodes and $66.6 \%$ of urinary tract infections caused

\begin{tabular}{|c|c|c|}
\hline & Susceptible & Resistant \\
\hline Ampicillin ${ }^{* *}$ & $2(14.3 \%)$ & $10(71.4 \%$ \\
\hline Amoxicillin-clavulin $^{\dagger}$ & $10(71.4 \%)$ & $2(14.3 \%)$ \\
\hline Piperacillin-tazobactam $\$$ & $11(78.5 \%)$ & $0(0 \%)$ \\
\hline Cefazolin ${ }^{* t}$ & $8(57.1 \%)$ & $3(21.4 \%)$ \\
\hline Ciprofloxacin & $2(14.3 \%)$ & $12(85.7 \%$ \\
\hline Gentamicin & $11(78.6 \%)$ & $3(21.4 \%)$ \\
\hline Trimethoprin-sufamethoxazole ${ }^{*}$ & $7(50 \%)$ & $7(50 \%)$ \\
\hline \multicolumn{3}{|c|}{$\begin{array}{l}\text { Rates presented as percentages of } 14 \text { samples tested for each antibiotic. "Indicates } \\
\text { presence of intermediate strain; "Indicates one strain not tested for susceptibility and } \\
\text { resistance; 'Indicates } 2 \text { strains not tested for susceptibility and resistance; 'Indicates three } \\
\text { strains not tested for susceptibility and resistance. }\end{array}$} \\
\hline
\end{tabular}

by ciprofloxacin-resistant microbes. Carigan and colleagues found that the incidence of post-biopsy sepsis increased from $0.71 \%$ in $2002-2003$ to $2.15 \%$ in $2010-2011 .^{1}$ There are other North American reports demonstrating a rising trend in post-TRUS biopsy complications. ${ }^{8}$ In a large Ontario-based study, Nam and colleagues found a 4-fold rise in hospital admission rates after TRUS-PB over a 10-year study period. ${ }^{2}$ American data reveal an increasing trend in post-biopsy infections from $0.4 \%$ to $1.1 \%$ between 1991 and $2007 .{ }^{3}$ In the context of rising antibacterial resistance, it is not surprising to notice an increased incidence of post-biopsy infections at our institution.

The concerning finding in our study is the emergence of bacteria not commonly implicated in post-biopsy sepsis. As other studies have identified, E. coli and Klebsiella species were the most commonly found bacteria in post-TRUS sepsis. ${ }^{8}$ However, Comamonas species are gram-negative ubiquitous aerobic rods, which were never documented to cause sepsis in Canada. This bacterial species has only been reported in few case reports globally. ${ }^{28}$ This isolated case of sepsis was observed in a patient with extensive travel history to India 3 months prior to biopsy. There is evidence to suggest that there is increased colonization with antibiotic resistant commensal bacteria after travelling to highly endemic regions of Asia; these microbes persist in the bowel for up to 6 months post-travel. ${ }^{6}$ In a study by Patel and colleagues, travel history has been shown to increase the risk

\begin{tabular}{|c|c|c|c|}
\hline & No. samples tested & Susceptibility & Resistance \\
\hline Ampicillin & 15 & $2(13.3 \%)$ & $12(80 \%)^{*}$ \\
\hline Amoxicillin-clavulin & 14 & $12(85.7 \%)$ & $2(14.3 \%)$ \\
\hline Cefazolin & 14 & $10(71.4 \%)$ & $3(21.4 \%)^{*}$ \\
\hline Piperacillin-tazobactam & 13 & $13(100 \%)$ & $0(0 \%)$ \\
\hline Ciprofloxacin & 16 & $2(12.5 \%)$ & $14(87.5 \%)$ \\
\hline Norfloxacin & 2 & $1(50 \%)$ & $1(50 \%)$ \\
\hline Gentamicin & 17 & $14(82.4 \%)$ & $3(17.6 \%)$ \\
\hline Trimethoprim-sulfamethoxazole & 17 & $9(52.9 \%)$ & $8(47.1 \%)$ \\
\hline
\end{tabular}

Rates presented as percentages of total number of samples tested for each antibiotic. ${ }^{*}$ Indicates presence of one intermediate strain. 
of post-biopsy infections by 2.7 ; in addition, antibiotic exposure less than 4 weeks prior to TRUS biopsy increased the risk by $4 .{ }^{6}$ We therefore suggest conducting relevant travel history assessment prior to initiating pre-biopsy prophylaxis. Based on our results, FQ and amoxicillin antibiotics are not suitable for pre-biopsy prophylaxis, while gentamicin and piperacillin-tazobactam were the most effective in treating post-TRUS sepsis.

There have been many attempts to reduce the rate of post-TRUS-PB infectious complications. Adjunctive measures with pre-biopsy rectal swabs, enemas, and povidone iodine rectal cleanse have shown conflicting and clinically insignificant results. ${ }^{14,27,29}$ Currently, there are no concrete mitigating strategies to reduce the risk of post-TRUS-PB associated infections. Furthermore, there are no clinical practice guidelines on the management of post-biopsy infectious complications. ${ }^{8}$ Several U.S. centres have adopted a strategy to use a centre-specific tailored approach based on local antibacterial resistance patterns. ${ }^{8}$ However, the clinical and cost benefits of this approach have yet to be determined. ${ }^{30}$ In the interim, the most practical bedside approach at our centre is a patient-specific risk factor assessment with individualized antibacterial prophylaxis.

There are 2 proposed avenues to improve post-biopsy outcomes: (1) the modification and optimization of the prostate biopsy technique or (2) a change in the existing prophylaxis methodology. More and more, transperineal prostate biopsy has been conducted in men with more than 1 previously negative TRUS biopsy with persistently elevated PSA. ${ }^{31}$ In the meantime, individualized risk and benefit assessment, including patient comorbidities, life expectancy and suitability for treatment of potential underlying malignancy, should be carefully considered. The benefit of tailored culture directed antibacterial prophylaxis poses many methodological and logistical challenges. There is no established evidence to indicate the optimal laboratory screening technique and appropriate duration between prebiopsy screening and prostate biopsy. ${ }^{30,32}$

There is emerging evidence demonstrating the benefit of increasing the conventional ciprofloxacin regimen with aminoglycosides. ${ }^{33,34}$ The Canadian guidelines recommend the addition of intravenous aminoglycosides to FQ in centeres with a high post-biopsy UTI incidence. ${ }^{10}$ Earlier studies demonstrated the benefit of adding aminoglycosides to ciprofloxacin prophylaxis in centres with high FQ resistance. ${ }^{33,34}$ More recently, Adibi and colleagues conducted a randomized controlled trial demonstrating a decline in post-biopsy hospitalizations due to infections from $3.8 \%$ to $0.6 \%$ when intra-muscular (IM) gentamicin was added to the FQ regimen. ${ }^{33}$ With increased post-biopsy sepsis caused by highly FQ resistant microbes, we could explore the benefit of using aminoglycosides to improve the antibacterial prophylaxis or formalin disinfection of prostate biopsy needles. ${ }^{35}$
The major limitation of our study is its retrospective design. We were unable to determine the medication compliance rate for the prescribed pre-biopsy regimen. Additionally, we may not have captured patients who potentially suffered from infections complications and never presented to the emergency department or an urgent care facility to seek medical attention.

The strength of our study is the inclusive nature of our data as this is the only centre, in a city of over 1 million people, performing TRUS-PB and all hospitals and urgent care facilities in the city had data available through their electronic medical records.

\section{Conclusions}

Our results suggest that the incidence of sepsis due to $\mathrm{FQ}$ resistant organisms increases following TRUS-PB compared to other centres in Canada. This finding is attributed to increasing local and global resistance to FQ antibiotics. The current antimicrobial prophylactic regimen requires reevaluation, and novel approaches need to be investigated. While we explore the alternatives, our recommendation is for urologists to conduct a thorough pre-biopsy risk factor assessment with tailored prophylaxis prior to prostate biopsy.

Competing interests: Dr. Rudzinski declares no competing financial or personal interests. Dr. Kawakami has received travel fees and sponsorship from Pentapharm and Baxter.

This paper has been peer-reviewed.

\section{References}

1. Carigan A, Roussy JF, Lapointe V, et al. Increasing risk of infectious complications after trans rectal ultrasound-guided prostate biopsies: Time to reassess antibacterial prophylaxis? Eur Urol 2012;62:453-9. http://dx.doi.org/10.1016/i.eururo.2012.04.044

2. Nam RK, Saskin R, Lee Y, et al. Increasing hospital admission rates for urological complications after transrectal ultrasound guided prostate biopsy. J Urol 2010;183:963-8. http://dx.doi.org/10.1016/i. juro.2009.11.043

3. Loeb S, Carter HB, Berndt SI, et al. Complications after prostate biopsy: Data from SEER-Medicare. J Urol 2011;186:1830-4. http://dx.doi.org/10.1016/i.juro.2011.06.057

4. Young JL, Liss MA, Szabo RJ. Sepsis due to fluoroquinolone-resistant Escherichia coli after transrectal ultrasound-guided prostate needle biopsy. J Urol 2009;74:332-8. http://dx.doi.org/10.1016/i.urology.2008.12.078

5. Feliciano J, Teper E, Ferrandino $M$, et al. The incidence of fluoroquinolone resistant infections after prostate biopsy - are fluoroquinolones still effective prophylaxis? J Urol 2008; 179:952-5. http:// dx.doi.org/10.1016/i.juro.2007.10.071

6. Patel U, Dasgupta $\mathrm{P}$, Amoroso $\mathrm{P}$, et al. Infection after transrectal ultrasonography-guided prostate biopsy: increased relative risks after recent international travel or antibiotic use. BJU Int 2012;109(12):1781-5. http://dx.doi.org/10.1111/j.1464-410X.2011.10561.x

7. Raaijmakers R, Kirkels WJ, Roobol MJ, et al. Complication rates and risk factors of 5802 transrectal ultrasound-guided sextant biopsies of the prostate within a population-based screening program. Urology 2002;60:826-30. http://dx.doi.org/10.1016/S0090-4295(02)01958-1

8. Williamson DA, Barrett LK, Rogers BA, et al. Infectious Complications Following Transrectal UltrasoundGuided Prostate Biopsy: New Challenges in the Era of Multidrug-Resistant Escherichia coli. Clin Infect Dis 2013;57:267-74. http://dx.doi.org/10.1093/cid/cit193 
9. Wolf JS JI, Bennett CJ, Dmochowski RR, et al. Best practice policy statement on urologic surgery antimicrobial prophylaxis. J Urol 2008;179:1379-90. http://dx.doi.org/10.1016/i.juro.2008.01.068

10. El-Hakim A, Moussa S. CUA guidelines on prostate biopsy methodology. Can Urol Assoc J 2010;4:89-94. http://dx.doi.org/10.5489/cuaj.10021

11. Aron M, Rajeev TP, Gupta NP. Antibiotic prophylaxis for transrectal needle biopsy of the prostate: A randomized controlled study. BJU Int 2000;85:682-5. http://dx.doi.org/10.1046/j.1464410x.2000.00576.x

12. Sabbagh R, McCormack M, Peloquin F, et al. A prospective randomized trial of 1-day versus 3-day antibiotic prophylaxis for transrectal ultrasound guided prostate biopsy. Can J Urol 2004; 11:2216-9.

13. Ruiz J. Mechanism of resistance to quinolones: Target alterations, decreased accumulation and DNA gyrase protection. J Antimicrob Chemother 2003;51:1109-17. http://dx.doi.org/10.1093/jac/dkg222

14. Duplessis CA, Bavaro M, Simons MP, et al. Rectal cultures before transrectal ultrasound-guided prostate biopsy reduce post-prostatic biopsy infection rates. Urology 2012;79:556-61. http://dx.doi. org/10.1016/j.urology.2011.09.057

15. Liss MA, Peterson EM, Johnston B, et al. Prevalence of $S T 131$ among fluoroquinolone-resistant Escherichic coli obtained from rectal swabs before transrectal prostate biopsy. Urology 2013;81:548-55. http:// dx.doi.org/10.1016/i.urology.2012.10.056

16. Steensels D, Slabbaert K, De Wever L, et al. Fluoroquinolone-resistant E. coli in intestinal flora of patients undergoing transrectal ultrasound-guided prostate biopsy - should we reassess our practices for antibiotic prophylaxis? Clin Microbiol Infect 2012;18:575-81. http://dx.doi.org/10.1111/i.1469$0691.2011 .03638 . x$

17. Batura D, Rao GG, Nielsen PB. Prevalence of antimicrobial resistance in intestinal flora of patients undergoing prostatic biopsy: Implications for prophylaxis and treatment of infections after biopsy. BJU Int 2010;106:1017-20. http://dx.doi.org/10.1111/i.1464-410X.2010.09294.x

18. Church D, Pitout J, Gregson D. Microbiology Newsletter. 2008; Volumes 1,2,3.

19. Alberta Health Services. Calgary: Calgary Lab Services Microbiology Newsletter; 2013. http://www. calgarylabservices.com/files/HealthcareProfessionals/Micro_Antibiograms/Community.pdf. Accessed May 20, 2014

20. Bouchillon $S$, Hoban DJ, Badal $R$, et al. Fluoroquinolone resistance among gram-negative urinary tract pathogens: Global smart program results, 2009-2010. Open Microbiol J 2012;6:74-8. http://dx.doi. org/10.2174/1874285801206010074

21. Dalhoff A. Global fluoroquinolone resistance epidemiology and implications for clinical use. Inderdiscip Perspect Infect Dis 2012;2012:976273.

22. Rogers BA, Sidjabat HE, Paterson DL. Escherichia coli 025b-ST131: A pandemic, multiresistant, communityassociated strain. J Antimicrob Chemother 2011;66:1-14. http://dx.doi.org/10.1093/jac/dkq415

23. Loeb $S$, van den Heuvel $S$, Zhu $X$, et al. Infectious complications and hospital admissions after prostate biopsy in a European randomized trial. Eur Urol 2012;61:1110-4. http://dx.doi.org/10.1016/i. eururo.2011.12.058
24. Simsir A, Kismali E, Mammadov R, et al. Is it possible to predict sepsis, the most serious complication in prostate biopsy? Urol Int 2010;84:395-9. http://dx.doi.org/10.1159/000296290

25. Levy MM, Fink MP, Marshall JC, et al. 2001 SCCM/ESICM/ACCP/ATS/SIS International Sepsis Definition Conference. Crit Care Med 2003;31:1250-6. http://dx.doi.org/10.1097/01. CCM.0000050454.01978.3B

26. Canadian Cancer Society's Advisory Committee on Cancer Statistics. Canadian Cancer Statistics 2013. Toronto, ON: Canadian Cancer Society; 2013.

27. Abugosh Z, Margolick J, Goldenberg LS, et al. A prospective randomized trial of povidone-iodine prophylactic cleansing of the rectum before the transrectal ultrasound guided prostate biopsy. J Urol 2013;189:132631. htrp://dx.doi.org/10.1016/i.juro.2012.09.121

28. Farshad $S$, Norouzi $F$, Aminshahidi $M$, et al. Two cases of bacteremia due to an unusual pathogen, Comamonas testosteroni in Iran and a review literature. J Infect Dev Ctries 2012;6:521-5.

29. Zani EL, Clark OA, Rodrigues Netto N Jr. Antibiotic prophylaxis for transrectal prostate biopsy. Cochrane Database Syst Rev 2011:5:CD006576.

30. Taylor AK, Zembower TR, Nadler RB, et al. Targeted antimicrobial prophylaxis using rectal swab cultures in men undergoing transrectal ultrasound guided prostate biopsy is associated with reduced incidence of postoperative infectious complications and cost of care. J Urol 2012;187:1275-9. http://dx.doi. org/10.1016/i.juro.2011.11.115

31. Mabjeesh NJ, Lidawi $G$, Chen J, et al. High detection rate of significant prostate tumors in anterior zones using transperitoneal ultrasound-guided template saturation biopsy. BJU Int 2012;1 10:993-7. http:// dx.doi.org/10.1111/i.1464-410X.2012.10972.x

32. Liss MA, Peeples AN, Peterson EM. Detection of fluoroquinolone-resistant organisms from rectal swabs by use of selective media prior to a transrectal prostate biopsy. J Clin Microbiol 2011;49:1116-8. http:// dx.doi.org/10.1128/JCM.01885-10

33. Adibi $M$, Hornberger $B$, Bhat $D$, et al. Reduction in hospital admission rates due to post-prostate biopsy infections after augmenting standard antibiotic prophylaxis. J Urol 2013;189:535-40. http://dx.doi. org/10.1016/i.juro.2012.08.194

34. Ho HS, Ng LG, Tan YH, et al. Intramuscular gentamicin improves the efficacy of ciprofloxacin as an antibiotic prophylaxis for transrectal prostate biopsy. Ann Acad Med Sing 2009;38:212-6.

35. Issa MM, Al-Qassab UA, Hall J, et al. Formalin disinfection of biopsy needle minimizes the risk of sepsis following prostate biopsy. J Urol 2013;190:1769-75. http://dx.doi.org/10.1016/i.juro.2013.04.134

Correspondence: Dr. Jan Krzysztof Rudzinski, Faculty of Medicine, University of Calgary, Calgary, AB; janı@ualberta.ca 